\title{
Quark matter nucleation with a microscopic hadronic equation of state
}

\author{
Domenico Logoteta, Constança Providência, Isaac Vidaña \\ Centro de Física Computacional, Department of Physics, \\ University of Coimbra, PT-3004-516 Coimbra, Portugal \\ Ignazio Bombaci \\ Dipartimento di Fisica "Enrico Fermi", Universitá di Pisa, \\ and INFN Sezione di Pisa, Largo Bruno Pontecorvo 3, I-56127 Pisa, Italy
}

\begin{abstract}
The nucleation process of quark matter in cold $(T=0)$ stellar matter is investigated using the microscopic Brueckner-Hartree-Fock approach to describe the hadronic phase, and the MIT bag model, the Nambu-Jona-Lasinio, and the Chromo Dielectric models to describe the deconfined phase of quark matter. The consequences of the nucleation process for neutron star physics are outlined. Hyperonic stars are metastable only for some of the quark matter equations of state considered. The effect of an hyperonic three body force on the metastability of compact stars is estimated, and it is shown that, except for the Nambu-Jona-Lasinio model and the MIT bag model with a large bag pressure, the other models predict the formation of hybrid stars with a maximum mass not larger than $\sim 1.62 M_{\odot}$.
\end{abstract}

PACS number(s): 97.60.-s, 97.60.Jd, 26.60.Dd, 26.60.Kp

\section{INTRODUCTION}

Quark matter (QM) nucleation in neutron stars has been studied by many authors both at zero [1 $[9]$ and finite temperature [11 16], due to its potential connection with explosive astrophysical events such as supernovae and gamma ray bursts. In all these works, the hadronic phase was described using phenomenological models, such as e.g., the well-known relativistic mean field (RMF) model based on effective Lagrangian densities where the baryon-baryon interaction is described in terms of meson exchanges [17]. Among the different RMF models, one of the most popular parametrizations is the one of Glendenning and Moszkowski [18] of the non-linear Walecka model which have been widely used to study the effect of the hadronic equation of state (EoS) on the QM nucleation process. In particular, the effect of different hyperon couplings on the critical mass 1, 2] for pure hadronic stars (HSs, i.e. neutron stars in which no fraction of QM is present) and the stellar conversion energy 19] was studied in Ref. 7]. It was found that increasing the value of the hyperon coupling constants, increases the stellar metastability threshold mass and the value of the critical mass, thus making the formation of quark stars (QSs, i.e. hybrid stars or strange stars depending on the details of the EoS for quark matter used to model the phase transition) less likely. In that work, the hadronic phase was also described using the quarkmeson-coupling model 20], concluding, in that case, that the formation of a quark star was only possible with using a small value of the bag pressure. In all these works the MIT bag model [21] was used to describe the quark matter phase. In a recent work [10], two models that contain explicitly the chiral symmetry were applied to describe the quark phase, namely the Nambu-Jona-Lasinio (NJL) model [22] (see also 23, 24]) and the Chromo Dielectric model (CDM) 25, 26. It was shown there that it is very difficult to populate the quark star branch with the NJL model and, therefore, all compact stars would be pure hadronic stars in that case. On the contrary, with the CDM, both hadronic and quark star configurations can be formed.

In the present work we study the nucleation of quark matter using an hadronic EoS based on microscopic calculations. In particular, we employ two hadronic EoS based on microscopic Brueckner-Hartree-Fock (BHF) calculations of hypernuclear matter. The first one (hereafter called Model 1) is the recent parametrization provided by Schulze and Rijken [27] which uses the Argonne V18 nucleon-nucleon (NN) 28] supplemented by the microscopic three-body force (TBF) of Ref. 29] between nucleons (NNN), and the recent Nijmegen extended softcore ESC08b hyperon-nucleon (YN) potentials [30]. The second one (hereafter called Model 2) is based on our recent work of Ref. 31] where we used the Argonne V18 NN force and the Nijmegen soft-core NSC89 YN one 32] in a microscopic BHF calculation of hyperonic matter supplemented with additional simple phenomenological density-dependent contact terms, that mimic the effect of NNN, NNY and NYY TBFs, to establish numerical lower and upper limits to the effect of hyperonic TBF on the maximum mass of neutron stars. To describe the quark phase, in the present work, we use the three different models already mentioned, the MIT bag model [21], the NJL model 22] and the CDM model [25].

The paper is organized in the following way. In sections II and III we briefly review the BHF approach and the main features of quark matter nucleation in hadronic stars, respectively. Our results are presented in Sec. IV. Finally, a summary and the main conclusions of this work are given in Sec. $\mathrm{V}$. 


\section{THE BHF APPROACH}

The BHF approach is the lowest order of the Brueckner-Bethe-Goldston (BBG) many-body theory [33]. In this theory, the ground state energy of nuclear matter is evaluated in terms of the so-called hole-line expansion, where the perturbative diagrams are grouped according to the number of independent hole-lines. The expansion is derived by means of the in-medium twobody scattering $G$ matrix. The $G$ matrix, which takes into account the effect of the Pauli principle on the scattered particles and the in-medium potential felt by each nucleon, has a regular behavior even for short-range repulsions, and it describes the effective interaction between two nucleons in the presence of a surrounding medium. In the BHF approach, the energy is given by the sum of only two-hole-line diagrams including the effect of two-body correlations through the $G$ matrix. It has been shown by Song et al. 34] that the contribution to the energy from three-hole-line diagrams (which account for the effect of three-body correlations) is minimized when the so-called continuous prescription [35] is adopted for the in-medium potential, which is a strong indication of the convergence of the hole-line expansion. The BHF approach has been extended to hyperonic matter by several authors [27, 31, 36]. The interested reader is referred to these works for the specific details of the BHF calculation of hyperonic matter, and to Ref. 33] for an extensive review of the BBG many-body theory.

\section{QUARK MATTER NUCLEATION IN HADRONIC STARS}

The conditions of phase equilibrium, in the case of a first-order phase transition [37], are given by the Gibbs' phase rule, which in the case of cold $(T=0)$ matter can be written as:

$$
P_{H}=P_{Q} \equiv P_{0} \quad \mu_{H}\left(P_{0}\right)=\mu_{Q}\left(P_{0}\right)
$$

where

$$
\mu_{H}=\frac{\epsilon_{H}+P_{H}}{n_{H}} \quad \mu_{Q}=\frac{\epsilon_{Q}+P_{Q}}{n_{Q}}
$$

are the Gibbs energies per baryon for the hadron $(\mathrm{H})$ and quark $(\mathrm{Q})$ phases, respectively, and the quantities $\epsilon_{H}\left(\epsilon_{Q}\right), P_{H}\left(P_{Q}\right)$, and $n_{H}\left(n_{Q}\right)$ denote respectively the total (i.e., including leptonic contributions) energy density, total pressure, and baryon number density of the two phases. Above the transition pressure $P_{0}$ the hadronic phase is metastable, and the stable quark phase will appear as a result of a nucleation process. Quantum fluctuations will form virtual drops of quark matter. The characteristic oscillation time $\nu_{0}^{-1}$ of these drops, in the potential energy barrier separating the metastable hadronic phase and quark phase, is set by strong interactions, which are responsible of the deconfinement transition, thus $\nu_{0}^{-1} \sim 10^{-23}$. This time is many orders of magnitude smaller than the weak interaction characteristic time $\left(\tau_{\text {weak }} \sim 10^{-8} \mathrm{~s}\right)$, consequently quark flavor must be conserved forming a virtual drop of quark matter. We call $Q^{*}$-phase this deconfined quark matter, in which the flavor content is equal to that of the $\beta$-stable hadronic phase at the same pressure and temperature. Soon after a critical size drop of quark matter is formed, the weak interactions will have enough time to act, changing the quark flavor fraction of the deconfined droplet to lower its energy, and a droplet of $\beta$-stable quark matter is formed. (hereafter the Q-phase).

This first seed of quark matter will trigger the conversion [19, 38, 39] of the pure hadronic star to a quark star. Thus, pure hadronic stars with values of the central pressure $P_{c}$ higher than $P_{0}$ and corresponding masses $M>M_{t h r} \equiv M\left(P_{0}\right)$, are metastable to the decay (conversion) to quark stars [1 8$]$. The mean lifetime of the metastable stellar configuration is related to the time needed to nucleate the first drop of quark matter in the stellar center, and it depends dramatically on the value of the stellar central pressure.

As in Refs. [1 3], we define as the critical mass $M_{c r}$ of the hadronic star sequence, the value of the stellar gravitational mass for which the nucleation time of a $Q^{*}$ matter droplet is equal to one year: $M_{c r} \equiv M_{H S}(\tau=$ $1 \mathrm{yr})$. Pure hadronic stars with $M>M_{c r}$ are thus very unlikely to be observed. $M_{c r}$ plays the role of an effective maximum mass [3] for the hadronic branch of compact stars.

In a cold and neutrino-free hadronic star the formation of the first drop of quark matter could take place solely via a quantum nucleation process. The basic quantity needed to calculate the nucleation time is the energy barrier separating the $\mathrm{Q}^{*}$-phase from the metastable hadronic phase. This energy barrier, which represents the difference in the free energy of the system with and without a $\mathrm{Q}^{*}$-matter droplet, can be written as [3, 40]

$$
U(\mathcal{R})=\frac{4}{3} \pi n_{Q^{*}}\left(\mu_{Q^{*}}-\mu_{H}\right) \mathcal{R}^{3}+4 \pi \sigma \mathcal{R}^{2},
$$

where $\mathcal{R}$ is the radius of the droplet (supposed to be spherical), and $\sigma$ is the surface tension for the surface separating the hadron from the $\mathrm{Q}^{*}$-phase. The energy barrier has a maximum at the critical radius $\mathcal{R}_{c}=2 \sigma /\left[n_{Q^{*}}\left(\mu_{H}-\mu_{Q^{*}}\right)\right]$.

The quantum nucleation time $\tau_{q}$ can be straightforwardly evaluated within a semi-classical approach [24, 40] and it can be expressed as

$$
\tau_{q}=\left(\nu_{0} p_{0} N_{c}\right)^{-1},
$$

where $p_{0}$ is the probability of tunneling the energy barrier $U(\mathcal{R})$ in its ground state, $\nu_{0}$ is the oscillation frequency of a virtual drop of the $\mathrm{Q}^{*}$-phase in the potential well, and $N_{c} \sim 10^{48}$ is the number of nucleation centers expected in the innermost part $\left(r \leq R_{n u c} \sim 100 \mathrm{~m}\right)$ of the hadronic star, where the pressure and temperature (in finite $\mathrm{T}$ case) can be considered constant and equal to their central values. 


\section{RESULTS AND DISCUSSION}

We will now discuss the results obtained with the two microscospic hadronic EoS considered and, in particular, we will comment whether the possible discussed scenarios are compatible with the recent measurement 41] of the mass of the pulsar PSR J1614-2230 with a mass $\mathrm{M}=$ $(1.97 \pm 0.04) M_{\odot} \cdot$

In Fig. 1 we plot the Gibbs energy per baryon as a function of pressure using the microscopic approach of Ref. [27] (Model 1) for the hadronic phase and one of the following models for the $Q^{*}$-phase: MIT bag (top panels), CDM (left bottom panel) and NJL (right bottom panel) model. It is interesting to note that the formation of the $Q^{*}$-phase is possible only in the case of the MIT bag model EoS with a low value of the bag constant $\left(B=85 \mathrm{MeV} \mathrm{fm}^{-3}\right)$. In all the other cases considered in Fig. 1, the curve for Gibbs energy per baryon for the $Q^{*}$-phase never crosses the one for the hadronic phase, consequently, the hadronic phase will always remain stable with respect to the formation of $Q^{*}$-phase droplets. For these three QM models, this result implies that the pure hadronic stars (hyperonic stars) described by Model 1 are stable up to their maximum mass configuration $M_{\max }^{H S}=1.37 M_{\odot}$.

By numerical integration of the TolmanOppenheirmer-Volkov equations 42], we have calculated the structural properties for pure hadronic and quark star sequences. The main results, in the case of Model 1 EoS for the hadronic phase, are summarized in Table [1. where we report the maximum gravitational mass $M_{\max }^{H S}$ (third column), the gravitational threshold mass $M_{t h r} \equiv M\left(P_{0}\right)$ for metastable configurations (fourth column), and the gravitational (baryonic) critical mass $M_{c r}$ $\left(M_{c r}^{b}\right)$ (fifth (sixth) column) for the pure hadronic star sequence. $M_{\text {fin }}$ (seventh column) is the gravitational mass of the hybrid star formed by the stellar conversion process of the HS with $M=M_{c r}$ and assuming baryon number conservation in the process [19] (i.e. assuming $\left.M_{f i n}^{b}=M_{c r}^{b}\right)$. Finally $E_{c o n v}$ is the total energy liberated in the stellar conversion. It is interesting to note that hybrid star configurations can be obtained with the MIT bag model with $B=85 \mathrm{MeV} \mathrm{fm}^{-3}$, and with the CDM. In the latter case, however, the transitory non- $\beta$-stable $Q^{*}$-phase is not energetically achievable (see left bottom panel Fig. 11). Thus in this case the hadronic star sequence is stable up to the maximum mass configuration (thus we have no entries in Tab. I for the quantities $M_{t h r}, M_{c r}, M_{c r}^{b}, M_{f i n}$ and $\left.E_{c o n v}\right)$. In the case of the MIT bag model with $B=85 \mathrm{MeV}$ $\mathrm{fm}^{-3}$ the $Q^{*}$-matter nucleation is possible and one has $M_{c r}=1.272 M_{\odot}$. The conversion of this star will produce an hybrid star with $M_{f i n}=1.233 M_{\odot}$. If this object is a member of a binary stellar system, eventual accretion of matter from the companion will allow it to reach a maximum mass of $1.544 M_{\odot}$. In this case the pulsar PSR J1614-2230 will neither be an hyperonic star nor an hybrid star.
We have also artificialy turned off the hyperonic degrees of freedom in Model $1 \mathrm{EoS}$, and considered pure nucleonic stars. In this case we have $M_{\max }^{H S}=2.27 M_{\odot}$, and a critical mass $M_{c r} \sim 2.2 M_{\odot}$ for all the quark matter EoS considered in Tab. I In all cases, however, the critical mass configuration will collapse to a black hole (BH entry in seventh column in Tab. I) and thus the hybrid star sequence can not be populated. In this case the pulsar PSR J1614-2230 would be an hadronic star containing only nucleons and leptons. We next discuss the results obtained with the hadronic EoS based on our recent work of Ref. [31] (Model 2) where, as we said, a microscopic Brueckner-Hartree-Fock approach of hyperonic matter based on the Argonne V18 NN and the NSC89 NY forces is supplemented with additional simple phenomenological density-dependent contact terms that mimic the effect of nucleonic and hyperonic threebody forces. In particular, we consider three different parametrizations of this model corresponding to different values of the incompressibility coefficient, $K_{\infty}$, of symmetric nuclear matter at saturation, and the parameter $x$, which characterizes the strengh of the hyperonic three-body forces: $P_{1}\left(K_{\infty}=236 \mathrm{MeV}\right.$ and $\left.x=1\right), P_{2}$ $\left(K_{\infty}=236 \mathrm{MeV}\right.$ and $\left.x=1 / 3\right)$ and $P_{3}\left(K_{\infty}=285 \mathrm{MeV}\right.$ and $x=1)$. The interested reader is referred to [31], and particularly to Tables 1 and 2 of this reference, for details. The results for this model are shown in Table II and Fig. 2 As in the previous case, we summarize in Table II the main stellar properties obtained with this model in combination with the MIT bag and the CDM. Note that in this case with the NJL model no transi-
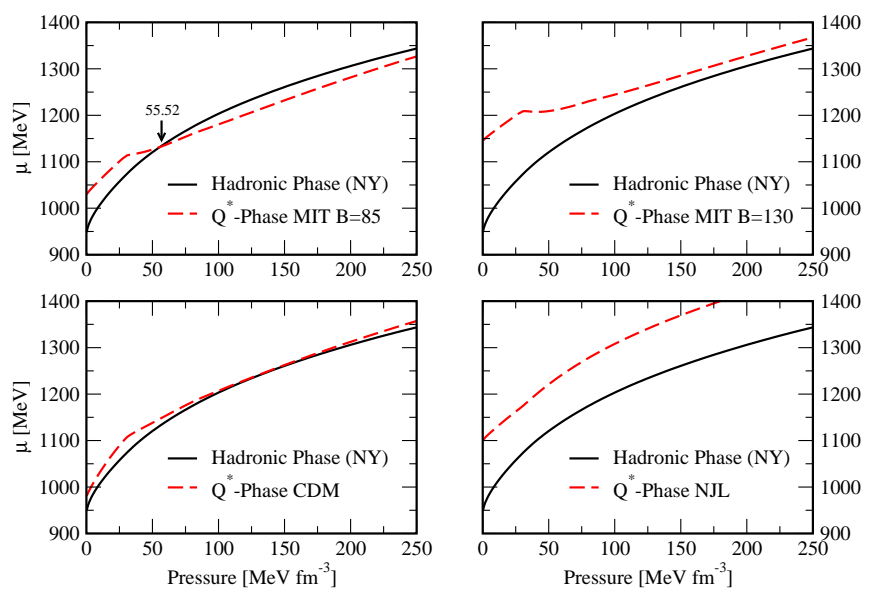

FIG. 1: (Color online) Gibbs energy per baryon at zero temperature as a function of the pressure for the hadronic phase (solid lines) and the $Q^{*}$-phase (dashed lines). Model 1 has been used to describe the hadronic phase. Results for the MIT bag model with $m_{u}=m_{d}=0, m_{s}=150 \mathrm{MeV}$ and two different values of the bag constant $B$, are presented in the top panels whereas those for the Chromo Dielectric (CDM) and the NJL models are shown in the bottom ones. The arrow and the corresponding number indicates the value (in $\mathrm{MeV} / \mathrm{fm}^{3}$ ) of the transition pressure $P_{0}$. 


\begin{tabular}{|c|c|c|c|c|c|c|c|c|}
\hline & & $M_{\max }^{H S}$ & $M_{t h r}$ & $M_{c r}$ & $M_{c r}^{b}$ & $M_{\text {fin }}$ & $E_{\text {conv }}$ & $M_{\max }^{H y b S}$ \\
\hline \multirow[t]{2}{*}{ nucleons+hyperons } & $\operatorname{MIT}(B=85)$ & 1.37 & 1.227 & 1.272 & 1.397 & 1.233 & 71.26 & 1.544 \\
\hline & CDM & 1.37 & - & - & - & - & - & 1.591 \\
\hline \multirow[t]{4}{*}{ only nucleons } & $\operatorname{MIT}(B=85)$ & 2.27 & 2.193 & 2.226 & 2.677 & $\mathrm{BH}$ & - & 1.544 \\
\hline & $\operatorname{MIT}(B=130)$ & 2.27 & 2.242 & 2.254 & 2.720 & $\mathrm{BH}$ & - & 1.471 \\
\hline & NJL & 2.27 & 2.229 & 2.246 & 2.708 & $\mathrm{BH}$ & - & 1.879 \\
\hline & CDM & 2.27 & 2.242 & 2.255 & 2.722 & $\mathrm{BH}$ & - & 1.592 \\
\hline
\end{tabular}

TABLE I: Stellar properties for the hadronic EoS of Ref. 27] (Model 1) in the case nuclear matter (only nucleons) or hyperonic matter (nucleons+hyperons) and different models for the quark phase. $M_{\max }^{H S}$ is the gravitational maximum mass of the pure hadronic star sequence, $M_{t h r} \equiv M\left(P_{0}\right)$ is the gravitational threshold mass for metastable stellar configurations, $M_{c r}\left(M_{c r}^{b}\right)$ the gravitational (baryonic) critical mass, $M_{\text {fin }}$ is the mass of the hybrid star which is formed by the stellar conversion of the hadronic star with $M=M_{c r}$ and assuming baryon number conservation in the process (i.e. $M_{\text {fin }}^{b}=M_{c r}^{b}$ ), finally $E_{c o n v}$ is the total energy, in unit of $10^{51} \mathrm{erg}$, liberated in the stellar conversion. All stellar masses are expressed in units of the solar mass $M_{\odot}=1.9889 \times 10^{33} \mathrm{~g}$. The bag pressure $B$ is given in $\mathrm{MeV} / \mathrm{fm}^{3}$

\begin{tabular}{|c|c|c|c|c|c|c|c|c|}
\hline & & $M_{\max }^{H S}$ & $M_{t h r}$ & $M_{c r}$ & $M_{c r}^{b}$ & $M_{f i n}$ & $E_{\text {conv }}$ & $M_{\max }^{H y b S}$ \\
\hline$P_{1}$ & MIT $B=85$ & 1.48 & 1.245 & 1.368 & 1.526 & 1.335 & 60.32 & 1.574 \\
\hline$P_{2}$ & MIT $B=85$ & 1.38 & 1.210 & 1.230 & 1.356 & 1.203 & 47.62 & 1.574 \\
\hline$P_{3}$ & MIT $B=85$ & 1.60 & 1.293 & 1.504 & 1.688 & 1.456 & 85.57 & 1.574 \\
\hline$P_{3}$ & CDM & 1.60 & 1.397 & 1.472 & 1.648 & 1.440 & 56.96 & 1.624 \\
\hline
\end{tabular}

TABLE II: Same as in Tab. I, but now for the hadronic EoS of Ref. 31] (Model 2) and three different parametrizations of this model: $P_{1}\left(K_{\infty}=236 \mathrm{MeV}\right.$ and $\left.x=1\right), P_{2}\left(K_{\infty}=236 \mathrm{MeV}\right.$ and $\left.x=1 / 3\right)$ and $P_{3}\left(K_{\infty}=285 \mathrm{MeV}\right.$ and $\left.x=1\right)$.

tion occurs for any of the three parametrizations $P_{1}, P_{2}$ and $P_{3}$. In Fig. 2 we plot the Gibbs energy per baryon as a function of pressure using: the MIT bag model for the $Q^{*}$-phase and an hadronic parametrization with the incompressibility $K_{\infty}=236 \mathrm{MeV}\left(P_{1}\right)$ and $K_{\infty}=285$ $\operatorname{MeV}\left(P_{3}\right)$ with $x=1$ in both cases. Results for the parametrization $P_{2}$, and the CDM and NJL models are not shown for conciseness. It is interesting to note that the parameter $x$, does not influence much the mass and radius of the hybrid star maximum mass configurations.

Note also (see Table II) that, similarly to Model 1, for the parametrizations $P_{1}$ and $P_{2}$, the formation of the $Q^{*}$-phase is possible only in the case of the MIT bag model EoS with a low value of the bag constant $(B=85$ $\mathrm{MeV} \mathrm{fm}^{-3}$ ). Nevertheless, for the parametrization $P_{3}$, the stable hybrid sequence may be populated from the stellar conversion of the critical mass hadronic star if the quark phase is described either with the MIT bag model (with $B=85 \mathrm{MeV} / \mathrm{fm}^{3}$ ) or with the CDM model. As already said, no transition is found for the NJL model. We also note that in the case of the parametrization $P_{3}$ plus the MIT bag model with $B=130 \mathrm{MeV} / \mathrm{fm}^{3}$ the $Q^{*}$ phase nucleation time at the center of the maximum mass $\left(M_{\text {max }}^{H S}\right)$ hadronic star is much larger than the age of the Universe, and thus, it is extremely unlikely to populate the hybrid star branch in this case. In the most favorable scenario the possible largest star mass would be $1.624 M_{\odot}$ (for CDM). This mass could occur if after, the conversion, the star accretes mass from an eventual companion star if the object is in a binary system.

\section{SUMMARY AND CONCLUSIONS}

Using the microscopic Brueckner-Hartree-Fock approach to describe the EoS of dense hadronic matter we have studied the possibility of occurrence of a deconfinement phase transition into quark matter in neutron star cores. Quark matter has been described with three different models, namely, the MIT bag, the CDM and the NJL models. We have concluded that hyperonic hadronic stars will not suffer a deconfinement phase transition except if the quark EOS is obtained using the MIT bag model with a value of the bag pressure $B=85 \mathrm{MeV} / \mathrm{fm}^{3}$. In this case, however, it is not possible to get a star with a mass above $1.54 M_{\odot}$. On the other hand, we have found that if the hadronic matter has no hyperons then deconfinement will occur only in very massive stars, with $M>2.2 M_{\odot}$ and the stars will decay into a black-hole. Within this microscopic approach to the hadronic phase the pulsar PSR J1614-2230 would be an hadronic star containing only nucleons and leptons. 
We have also studied the possible effect of a hyperonic TBF using the model proposed in [31]. It was shown that for the hardest EOS with $K_{\infty}=285 \mathrm{MeV}$ and $x=1$ an hybrid star could be formed. Only NJL and the MIT with $B=130 \mathrm{MeV} / \mathrm{fm}^{3}$ did not predict a metastable star in this case. Within this scenario a maximum mass of $1.624 M_{\odot}$ was predicted, very far from the mass of the PSR J1614-2230. However, it was also shown that it was not so much the hyperonic TBF strengh but more the incompressibility of the nucleonic part of the EoS that defines the possible deconfinement transition.

\section{Acknowledgments}

This work has been partially supported by the initiative QREN financed by the UE/FEDER throught the Programme COMPETE under the projects PTDC/FIS/113292/2009 and CERN/FP/116366/2010, the grant $\mathrm{SFRH} / \mathrm{BD} / 62353 / 2009$, and by COMPSTAR, an ESF Research Networking Programme.
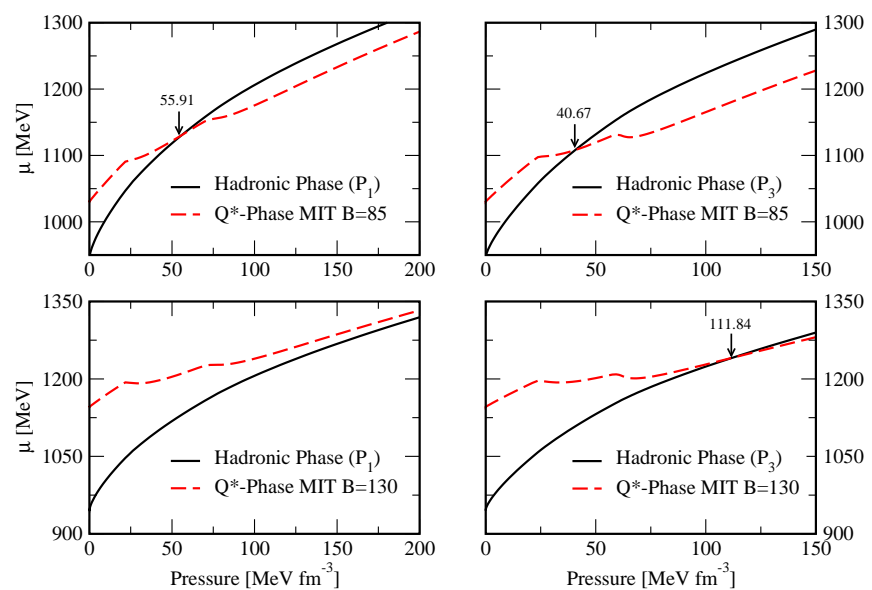

FIG. 2: (Color online) Gibbs energy per baryon at zero temperature as a function of the pressure for the hadronic phase (solid lines) and the $Q^{*}$-phase (dashed lines). The parametrizations $P_{1}$ (left panels) and $P_{3}$ (right panels) of the Model 2 introduced in [31] have been used to describe the hadronic phase. Results for the MIT bag model with $B=85$ (top panels) and $130 \mathrm{MeV} / \mathrm{fm}^{3}$ (bottom panels) are presented. The arrow and the corresponding number indicates the value (in $\mathrm{MeV} / \mathrm{fm}^{3}$ ) of the transition pressure $P_{0}$.

[1] Z. Berezhiani, I. Bombaci, A. Drago, F. Frontera, A. Lavagno, Nucl. Phys. B - Proceedings Supplements 113, 269 (2002).

[2] Z. Berezhiani, I. Bombaci, A. Drago, F. Frontera, and A. Lavagno, Astrophys. J. 586, 1250 (2003).

[3] I. Bombaci, I. Parenti, and I. Vidaña, Astrophys. J. 614, 314 (2004).

[4] A. Drago, A. Lavagno, and G. Pagliara, Phys. Rev. D
69, 057505 (2004).

[5] G. Lugones and I. Bombaci, Phys. Rev. D 72, 065021 (2005).

[6] I. Bombaci, G. Lugones, and I. Vidaña, Astron. and Astrophys. 462, 1017 (2007).

[7] I. Bombaci, P.K. Panda, C. Providência, and I. Vidaña, Phys. Rev. D 77, 083002 (2008).

[8] C. Bambi and A. Drago, Astropart. Phys. 29, 223 (2008). 
[9] A. Drago, G. Pagliara, and J. Schaffner-Bielich, J. Phys. G 35, 014052 (2008).

[10] D. Logoteta, I. Bombaci, C. Providência, and Isaac Vidaña, Phys. Rev. D 85, 023003 (2012).

[11] J. E. Horvath, O. G. Benvenuto, and H. Vucetich, Phys. Rev. D 45, 3865 (1992).

[12] J. E. Horvath, Phys. Rev. D 49, 5590 (1994).

[13] M. L. Olesen and J. Madsen, Phys. Rev. D 49, 2698 (1994).

[14] H. Heiselberg, in Strangeness and Quark Matter, Ed. G. Vassiliadis, World Scientific, 338 (1995); arXiv:hep-ph/9501374.

[15] I. Bombaci, D. Logoteta, P.K. Panda, C. Providência, and I. Vidaña, Phys. Lett. B 680, 448 (2009).

[16] I. Bombaci, D. Logoteta, C. Providência, and I. Vidaña, Astron. and Astrophys. 528, A71 (2011).

[17] B. D. Serot, and J. D. Walecka, Adv. Nucl. Phys. 16, (1986); Int. J. Mod. Phys. E 6, 515 (1997).

[18] N. K. Glendenning and S. A. Moszkowski, Phys. Rev. Lett. 67, 2414 (1991).

[19] I. Bombaci and B. Datta, Astrophys. J. 530, L69 (2000).

[20] P. A. M. Guichon, Phys. Lett. B 200, 235 (1988); K. Saito and A. W. Thomas, Phys. Lett. Bb 327, 9 (1994); P. A. M, Guichon, K. Saito, E. Rodionov, and A. W. Thomas, Nucl. Phys. A 601, 349 (1996); K. Saito, K. Tsushima, and A. W. Thomas, Nucl. Phys. A 609, 339 (1996); P. K. Panda, A. Mishra, J. M. Eisenberg, and W. Greiner, Phys. Rev. C 56, 3134 (1997).

[21] E. Farhi and R. L. Jaffe, Phys. Rev. D 30, 2379 (1984).

[22] Y. Nambu and G. Jona-Lasinio, Phys Rev. 122, 345 (1961).

[23] M. Buballa, Phys. Rep. 407, 205 (2005).

[24] D.P. Menezes and C. Providência, Phys. Rev. C 68, 035804 (2003); M. Baldo, M. Buballa, G. F. Burgio, F. Neumann, M. Oertel, and H.-J. Schulze Phys. Lett. B 562, 153 (2003).

[25] H. J. Pirner, G. Chanfray, and O. Nachtmann, Phys. Lett. B 147, 249 (1984).

[26] J. A. Mcgovern, M. C. Birse, and D. Spanos, J. Phys. G 16, 1561 (1990); W. Broniowski, M. Cibej, and M. Kutschera and M. Rosina, Phys Rev. D 41, 285 (1990); S. K. Gosh and S. C. Pathak, J. Phys. G 18, 755 (1992); T. Neuber, M. Fiolhais, K. Goeke, and J. N. Urbano, Nucl.Phys. A 560, 909 (1993).

[27] H.-J. Schulze and T. Rijken, Phys. Rev. C 84, 035801 (2011).

[28] R. B. Wiringa, V. G. J. Stoks, and R. Schiavilla, Phys. Rev. C, 51, 38 (1995).

[29] Z. H. Li, U. Lombardo, H.-J. Schulze, and W. Zuo, Phys. Rev. C 77, 034316 (2008).
[30] T. Rijken, M. Nagels, and Y. Yamamoto, Nucl. Phys. A 835, 160 (2010); Y. Yamamoto, E. Hiyama, and T. Rijken, ibid. 835, 350 (2010); T. Rijken, M. Nagels, and Y. Yamamoto, Prog. Theor. Phys. Suppl. 185, 14 (2010); Y. Yamamoto, T. Motoba, and T. Rijken, ibid. 185, 72 (2010).

[31] I. Vidaña, D. Logoteta, C. Providência, A. Polls, and I. Bombaci, EPL 94, 11002 (2011).

[32] P. M. M. Maessen, Th. A. Rijken, and J. J. de Swart, Phys. Rev. C 40, 2226 (1989).

[33] B. D. Day, Rev. Mod. Phys. 39, 719 (1967); M. Baldo, in Nuclear Methods and the Nuclear Equation of State (World Scientific, Singapore, 1999); M. Baldo, and G. F. Burgio, Reports on Progress in Physics, Volume 75, Issue 2, pp. 026301 (2012).

[34] H. Q. Song, M. Baldo, G. Giansiracusa, and U. Lombardo, Phys. Rev. Lett. 81, 1584 (1998); Phys. Lett. B 411, 237 (1999).

[35] J. P. Jeneuke, A. Lejeune, and C. Mahaux, Phys. Rep. 25, 83 (1976).

[36] H.-J. Schulze, A. Lejeune, J. Cugnon, M. Baldo, and U. Lombardo, Phys. Lett. B 355, 21 (1995); H-.J. Schulze, M. Baldo, U. Lombardo, J. Cugnon, and A. Lejeune, Phys. Rev. C 57, 704 (1998); M. Baldo, G. F. Burgio, and H.-J. Schulze, Phys. Rev. C 58, 3688 (1998); I. Vidaña, A. Polls, A. Ramos, and V. G. J. Stoks, Phys. Rev. C 61, 025802 (2000); M. Baldo, G. F. Burgio, and H.-J. Schulze, Phys. Rev. C 61, 055801 (2000); I. Vidaña, A. Polls, A. Ramos, L. Engvik, and M. Hjorth-Jensen, Phys. Rev. C, 62, 035801 (2000) 035801; H.-J. Schulze, A. Polls, A. Ramos, and I. Vidaña, Phys. Rev. C, 73 (2006) 058801.

[37] The deconfinement transition in the high density region relevant for neutron stars is assumed to be of first order.

[38] A. V. Olinto, Phys. Lett. B 192, 71 (1987).

[39] H. Heiselberg, G. Baym, and C. J. Pethick, Nucl. Phys. B (Proc. Suppl.) 24, 144 (1991).

[40] K. Iida and K. Sato, Phys. Rev. C 58, 2538 (1998).

[41] P. B. Demorest, T. Pennucci, S. M. Ransom, H. S. E. Roberts, and J. W. T. Hessel, Nature 467, 1081 (2010).

[42] S. L. Shapiro and S. A. Teukolsky, Black Holes, White Dwarfs and Neutron Stars (Wiley, New York, 1983); N. K. Glendenning, Compact Stars: Nuclear Physics, Particle Physics and General Relativity, 2nd ed. (Springer, Berlin, 2000); P. Haensel, A. Y. Potekhin, and D. G. Yakovlev, Neutron Stars 1: Equation of State and Structure (Astrophysics and Space Science Library, Springer, New York, 2007). 